\title{
In planta and in silico characterization of five sesquiterpene synthases from Vitis vinifera (cv. Shiraz) berries
}

Dueholm, Bjørn; Drew, Damian P.; Sweetman, Crystal; Simonsen, Henrik T.

Published in:

Planta

Link to article, DOI:

10.1007/s00425-018-2986-7

Publication date:

2019

Document Version

Peer reviewed version

Link back to DTU Orbit

Citation (APA):

Dueholm, B., Drew, D. P., Sweetman, C., \& Simonsen, H. T. (2019). In planta and in silico characterization of five sesquiterpene synthases from Vitis vinifera (cv. Shiraz) berries. Planta, 249(1), 59-70.

https://doi.org/10.1007/s00425-018-2986-7

\section{General rights}

Copyright and moral rights for the publications made accessible in the public portal are retained by the authors and/or other copyright owners and it is a condition of accessing publications that users recognise and abide by the legal requirements associated with these rights.

- Users may download and print one copy of any publication from the public portal for the purpose of private study or research.

- You may not further distribute the material or use it for any profit-making activity or commercial gain

- You may freely distribute the URL identifying the publication in the public portal 
In planta and in silico characterization of five sesquiterpene synthases from Vitis vinifera (cv. Shiraz) berries

Bjørn Dueholm $^{\mathrm{a}^{*}}$, Damian P Drew ${ }^{\mathrm{a}, \mathrm{b}^{*}}$, Crystal Sweetman ${ }^{\mathrm{b}}$, Henrik T Simonsen ${ }^{\mathrm{a} \dagger}$

a. Department of Biotechnology and Biomedicine, Technical University of Denmark, DK-2800 Lyngby, Denmark.

b. Wine Science, School of Agriculture, Food and Wine, University of Adelaide, Urrbrae SA 5064, Australia.

* These authors contributed equally to the work.

† Corresponding author: hets@dtu.dk 


\section{Abstract}

Main conclusion: Five Vitis vinifera sesquiterpene synthases were characterized, two previously uncharacterized, one being a caryophyllene/cubebene synthase and the other a cadinene synthase. Residue differences with other Vitis sesquiterpene synthases are described.

The biochemical composition of grape berries at harvest can have a profound effect on the varietal character of the wine produced. Sesquiterpenes are an important class of volatile compounds produced in grapes that contribute to the flavor and aroma of wine, making the elucidation of their biosynthetic origin an important field of research. Five cDNAs corresponding to sesquiterpene synthase genes (TPSs) were isolated from Shiraz berries and expressed in planta in Nicotiana benthamiana followed by chemical characterization by GCMS. Three of the TPS cDNAs were isolated from immature berries and two were isolated from ripe Shiraz berries. Two of the investigated enzymes, TPS26 and TPS27, have been previously investigated by expression in E. coli, and the in planta products generally correspond to these previous studies. The enzyme TPS07 differed by eight amino acids (none of which are in the active site) from germacrene B and D synthase isolated from Gewürztraminer grapes and characterized in vitro. Here in planta characterization of VvShirazTPS07 yielded ylangene, germacrene $\mathrm{D}$ and several minor products. Two of the enzymes isolated from immature berries were previously uncharacterized enzymes. VvShirazTPS-Y1 produced cadinene as a major product and at least 17 minor sesquiterpenoid skeletons. The second, VvShirazTPS-Y2, was characterized as a caryophyllene/cubebene synthase, a combination of products not previously reported from a single enzyme. Using in silico methods, we identified residues that could play key roles regarding differences in product formation of these enzymes. The first ring-closure that is either a 1,10- or 1,11-ring closure is likely controlled by three neighboring amino acids in helices G1, H2, and J. As for many other investigated TPS enzymes, we also observe that only a few residues can account for radical changes in product formation.

Keywords: Sesquiterpene synthase, Shiraz, isoprenoid, sesquiterpenes, wine aroma 


\section{Introduction}

Terpenoids are the most diverse and abundant class of specialized metabolites in the plant kingdom and perform a variety of functions, including defense against insects and microbes (Paré and Tumlinson 1999) or the attraction of pollinators (Martin et al. 2009). Many terpenoids are relatively volatile and therefore have the potential to act as aroma and fragrance compounds (Zhan et al. 2014; Ikram et al. 2015). These volatile compounds are particularly relevant in the cultivated grapevine, due to the use of its berries in wine making (Lund and Bohlmann 2006; Dunlevy et al. 2009). There has been significant research into the effect of monoterpenoids such as linalool, nerol, and geraniol on the muscat aroma of white wine varieties (Sánchez-Palomo et al. 2005; Vilanova and Sieiro 2006; Dziadas and Jeleń 2010), along with the genetic basis for monoterpene biosynthesis in grapes (Martin and Bohlmann 2004; Battilana et al. 2011; Martin et al. 2012). Some more recent studies have also investigated the presence of sesquiterpenoids in grapes (Coelho et al. 2006; Kalua and Boss 2010; May and Wüst 2012; Matarese et al. 2013, 2014), although their presence in wine, and the individual and collective effect of sesquiterpenes on the aroma of wine is less understood (Robinson et al. 2014).

The first specialized metabolic step in the biosynthesis of sesquiterpenes is performed by a sesquiterpene synthase, a member of the larger terpene synthase (TPS) family of enzymes, which utilises farnesyl pyrophosphate to produce highly specialized 15-carbon structures. This metabolic step is often followed by subsequent metabolic steps performed by cytochrome P450 enzymes that 'decorate' the carbon backbone with different oxidations (alcohols, aldehydes, ketones, and carboxylic acids) (Weitzel and Simonsen 2015; Yang et al. 2015). The diversity of terpenoids is mainly due to the huge diversity within the terpene synthase gene family (Chen et al. 2011). In species such as Vitis vinifera, the large number of TPS coding sequences within the genome also influences the numerous aroma compounds produced (Wood et al. 2008; Martin et al. 2010; Ilc et al. 2017).

Here, we have characterized the biosynthetic products of five $V$. vinifera sesquiterpene synthases in Nicotiana benthamiana. The active site of sesquiterpene synthases are comprised of six of the $14 \alpha$-helices from the C-terminal domain as well as two loops (Degenhardt et al. 2009). At least one cyclization of the farnesyl/nerolidyl cation commonly takes place in the active site and the first cyclization, for instance a 1,10- or a 1,11-ring closure (Fig. 1), further guides the final product formation (Davis and Croteau 2000). Homology modeling and docking 
provide a means for identifying critical residues of an enzyme, given that an appropriate template (or templates) exists (Sali and Blundell 1993; Rupasinghe and Schuler 2006). As a crystal structure of a plant sesquiterpene synthase exists (Starks et al. 1997), we modeled the enzymes' active sites and performed docking of relevant intermediates to identify important residue differences that could play key roles in the cyclization of the farnesyl cation and/or nerolidyl cation.

\section{Materials and methods}

\section{Amplification of $V v T P S$ cDNAs}

Total RNA was isolated from grape berries of Vitis vinifera $\mathrm{cv}$. Shiraz. Berries were snap frozen in liquid nitrogen and stored at $-80{ }^{\circ} \mathrm{C}$ prior to RNA extraction. Total RNA was isolated as described (Sweetman et al. 2012) and cDNA synthesized using the iScript ${ }^{\mathrm{TM}}$ Select cDNA Synthesis Kit (Bio-Rad) according to the manufacturer's instructions. Primer pairs were designed for five TPS genes that have been shown to be actively expressed in maturing grape berries (Sweetman et al. 2012), with top strand primers incorporating a terminal 5' CACC motif to facilitate ligation into pENTR/D-TOPO (Invitrogen) vectors. cDNAs were amplified with Phusion ${ }^{\circledR}$ High-Fidelity DNA Polymerase (NEB) and at least 3 amplicons of each TPS were sequenced in the first instance (AGRF, Adelaide). To facilitate expression of TPS enzymes from plant-specific plasmids USER cloning was performed as described previously (NourEldin et al. 2006). In brief, primer pairs were designed with USER-overhangs to allow cloning of TPS cDNAs into a pEAQ-HT vector, harboring the viral p19 suppressor that had been modified to contain a USER insertion cassette. The five genes were submitted to NCBI with the following accession numbers: LOC100267145 for VvShirazTPS07, LOC100241877 for VvShirazTPS26, LOC100257265 for VvShirazTPS27, LOC100232954 for VvShirazTPS-Y1, and LOC100260629 for VvShirazTPS-Y2.

\section{In planta characterization of expressed TPS}

Nicotiana benthamiana plants were grown from seeds at $24{ }^{\circ} \mathrm{C}$ day/19 ${ }^{\circ} \mathrm{C}$ night for 5 weeks prior to infiltration with Agrobacterium tumefaciens containing the pEAQ-HT-TPS plasmids as per previously described methods (Bach et al. 2014). Approximately 48-72 hours post infiltration, whole leaf samples of transformed $N$. benthamiana were inserted into a $20 \mathrm{~mL}$ glass vial and extracted at $60{ }^{\circ} \mathrm{C}$ with a 57298-U, 50/30 $\mu \mathrm{m}$ divinylbenzene-carboxen- 
polydimethylsiloxane-stableFlex/SS (Supelco, Sigma-Aldrich, Denmark) fiber, which was exposed to the air in the vial for $20 \mathrm{~min}$ at $37^{\circ} \mathrm{C}$. Between samples, fibers were reconditioned by heating to $240{ }^{\circ} \mathrm{C}$ for $20 \mathrm{~min}$. Analysis was performed in triplicate for each sample and leaves expressing only the p19 suppressor from the pEAQ-HT vector were used as negative controls.

\section{GC-MS and data analysis}

Analyses were performed using a Shimadzu GC-MS-QP2010 equipped with a 29 m, $0.25 \mathrm{~mm}$ diameter $\times 0.25 \mu \mathrm{m}$ film thickness Agilent HP-5MS UI silica capillary column inserted directly into the ion source (Drew et al. 2012, 2013) at $16 \mathrm{kPa}$ and a column flow of $1.25 \mathrm{~mL} / \mathrm{min}$. The injection port temperature was $250{ }^{\circ} \mathrm{C}$, with lower temperatures tested in the first instance to ensure thermal degradation was not significant (Andersen et al. 2015). The oven temperature commenced at $60{ }^{\circ} \mathrm{C}$ for $3 \mathrm{~min}$, and increased to $160{ }^{\circ} \mathrm{C}$ at a rate of $7^{\circ} \mathrm{C} / \mathrm{min}$, then to $300{ }^{\circ} \mathrm{C}$ at $50{ }^{\circ} \mathrm{C} / \mathrm{min}$. This temperature was held for $5 \mathrm{~min}$ and then increased to $320^{\circ} \mathrm{C}$ at a rate of 50 ${ }^{\circ} \mathrm{C} / \mathrm{min}$, where it was maintained for $3 \mathrm{~min}$. The carrier gas was $\mathrm{H}_{2}$ and the ionization electron energy was $70 \mathrm{eV}$. The ion source temperature was $230{ }^{\circ} \mathrm{C}$ with an interface temperature 280 ${ }^{\circ} \mathrm{C}$. The total run time was 28.49 min. A C7-C30 standard series (Sigma-Aldrich, Denmark) was used to calculate retention indices (RI). All data were analyzed using the Shimadzu software Lab Solutions, GC-MS Solutions version 2.50 SU3. NIST and Wiley 2008 libraries were used in conjunction with the NIST Standard Reference Database for compound identification. Putative compound assignment was made based on a combination of RI and MS similarity, and by comparison to authentic standards for $\alpha$-guaiene, $\alpha$-bulnesene, $\alpha$-copaene, $\delta$ cadinene, $\alpha$-humulene, and $\beta$-caryophyllene. The distribution of products from the individual enzymes is given as a percentage of area of the total integrated area per run. This reflects directly to the total ion count per run.

\section{Homology modeling and active site volumes}

Only a few TPS enzymes from plants have been structurally determined (Starks et al. 1997; Greenhagen et al. 2006; Li et al. 2013). However, the overall structure is highly conserved between TPS enzymes across kingdoms (Gao et al. 2012), which enables the construction of reliable homology models. For generating homology models, the crystal structure of 5-epiaristolochene from $N$. benthamiana (TEAS, Worldwide Protein Data Bank entry 5EAT) was chosen. This template has few and minor gaps to the present VvShirazTPS targets and an 
identity to the targets between $44-47 \%$ (similarities $66-68 \%$ ). This template was also used as template in Drew et al. (2016) for VvGauS. Homology models for VvTPS07, VvTPS26, VvTPS27, VvTPS-Y1, and VvTPS-Y2 were created with MODELLER v9.17 (Webb and Sali 2016). The homology models were validated with MolProbity (Chen et al. 2010) and Verify3D (Lüthy et al. 1992).

Volumes of the active sites were measured with POVME 2.0.1 (Durrant et al. 2011, 2014) centered around the active site of the TEAS template [coordinates: 111, 40, 48] and a radius of the sphere set at $10 \AA$ as well as the removal of points that fall outside the convex hull of the active site.

\section{Docking}

The farnesyl cation and/or nerolidyl cation commonly undergoes at least one cyclization in the active sites of sesquiterpene synthases. Based on previous reports (Davis and Croteau 2000; Degenhardt et al. 2009), the product profiles of the five investigated $V$. vinifera $\mathrm{cv}$. Shiraz TPSs (VvShirazTPSs) are assumed to arise from an initial cyclization that is either a 1,10- or a 1,11ring closure (Fig. 1). Docking of relevant intermediates was performed with AutoDock Vina (Trott and Olson 2010). Modeling and docking was performed in UCSF Chimera v1.12 (Pettersen et al. 2004; Meng et al. 2006). 


\section{Results and discussion}

\section{Identification and characterization of $V$. vinifera terpene synthases}

The five enzymes, VvShirazTPS07, VvShirazTPS26, VvShirazTPS27, VvShirazTPS-Y1, and VvShirazTPS-Y2, were isolated from ripe and immature berries as previously described (Sweetman et al. 2012). N. benthamiana was used as an expression host to examine the five enzymes, and the volatiles were analyzed using HS-SPME-GC-MS. The results are shown in Table 1, and the GC-MS chromatograms can be found in the supplementary data set (Online Resource 1). The analysis showed that the terpene synthases can be assigned accordingly: VvShirazTPS07 is a ylangene/germacrene synthase, VvShirazTPS26 is a multiple-product synthase, VvShirazTPS27 is an isocaryophyllene synthase, VvShirazTPS-Y1 is a cadinene synthase, and VvShirazTPS-Y2 is a caryophyllene/cubebene synthase. As also seen in Table 1 , all of the enzymes produce a range of sesquiterpenes, which is also reflected in the volatile profiles found in $V$. vinifera. The farnesyl cation can undergo either 1,10- or 1,11-cyclization, giving rise to either an $(E, E)$-germacradienyl cation or an $(E, E)$-humulyl cation, respectively (Fig. 1). The farnesyl cation can also undergo isomerization to become a (Z,E)-germacradienyl cation via 1,10-ring closure as well as other cations via 1,6-, 1,7-, or 1,11-ring closure (Degenhardt et al. 2009; Christianson 2017).

\section{Active site investigations}

In order to understand the capacity of different enzymes to generate different products, the active sites were examined with state-of-the-art modeling technologies based on previous models, especially that of Nicotiana tabacum TEAS (Starks et al. 1997). The amino acid numbering in Fig. 2 is relative to the TEAS template. The TEAS crystal structure consists of $22 \alpha$-helices, of which 14 (helices A to K) are part of the C-terminal domain (Davis and Croteau 2000). Some of the helices are joined by loops of which some contain critical residues. Fulllength sequences can be seen in the supplementary data (Online Resource 2).

\section{A-C loop and helix C, aa 264-275}

Residues $\mathrm{Arg}^{264}$ and $\mathrm{Arg}^{266}$ in the A-C loop fold inward with the former being proximate to $\mathrm{C} 1$ of F(PP). Together with $\mathrm{Arg}^{441}$ in helix H2, these positively charged residues together with $\mathrm{Mg}^{2+}$ ions aid in directing the pyrophosphate moiety away from the hydrophobic pocket. These three arginine residues are conserved across all the investigated VvShirazTPS enzymes. As for 
TEAS, the tryptophan residue in helix C ( $\operatorname{Trp}^{273}$ in TEAS), which is also conserved across the investigated VvShirazTPS enzymes, is likely involved as a proton acceptor for the final deprotonation step. This residue, together with another aromatic residue in the J-K loop (Tyr ${ }^{527}$ in TEAS) is capable of engaging in cation- $\pi$ interactions (Dougherty 1996; Starks et al. 1997). Gly $^{281}$ in VvShirazTPS26 (Cys ${ }^{270}$ in TEAS) corresponds to Cys in the other investigated VvShirazTPS enzymes. For VvGuaS this residue is also Gly. The sulfhydryl group of the Cys residue in TEAS forms a hydrogen bond with $\mathrm{Y}^{527}$, which may aid in stabilizing the tyrosine residue in the first cyclization event, a stabilization that is not possible for VvShirazTPS26 and VvGauS.

Helix D and helix F, aa 294-305 and 360-385

Changing Thr to Phe in helix D of ZmTPS4 (from Zea mays) lead to inactivity with regards to (E,E)-FPP but retained activity with regards to (Z,E)-FPP (Köllner et al. 2006). The corresponding residue is Ile in VvShirazTPS07, -27, -Y1, and -Y2 (Ile ${ }^{294}$ in TEAS), Leu in VvShirazTPS26, and Phe in VvGauS. This residue may thus have an impact on isomerization of the farnesyl cation to the nerolidyl cation (Fig. 1). The polar threonine in VvShirazTPS-Y1, -Y2, and -27 in helix D (Ile ${ }^{297}$ in TEAS) corresponds to the hydrophobic Ile in VvShirazTPS07 and -26, and Leu in VvGauS. In the homology models, this residue is close to the C1 cation on the farnesyl/nerolidyl cation molecule after expulsion of the pyrophosphate group. This difference in polarity may have an impact on the deprotonation.

Site-directed mutagenesis of $\mathrm{Thr}^{296}$ (Ser ${ }^{298}$ in TEAS) in an amorpha-4,11-diene synthase (ADS) from Artemisia annua iterates the importance of this residue in the cyclization reaction, since substituting it with $\mathrm{Val}$ produces profiles of mainly linear products instead of cyclic (Fang et al. 2017). The equivalent residue is serine for all of the investigated VvShirazTPS enzymes. Further, mutants in which residue $\mathrm{Leu}^{374}\left(\mathrm{Tyr}^{376}\right.$ in TEAS) in helix $\mathrm{F}$ is substituted by larger residues (e.g. Tyr or Phe), the 1,10-ring closure of the bisabolyl cation (which is the second cyclization event in ADS) is moderately impaired and the mutants have aberrant product profiles, some being 1,11-ring closure products (Fang et al. 2017). Tyr is the corresponding residue all the investigated VvShirazTPS enzymes (Fig. 2). The DDxxD motif is part of helix D and plays a crucial role in binding the pyrophosphate group of FPP (Starks et al. 1997). 
Helices G1 and G2, aa 398-403 and 404-409

The active-site triad of threonine residues ( $\mathrm{Thr}^{401}-\mathrm{Thr}^{402}-\mathrm{Thr}^{403}$ ) in helix G1 of TEAS and HVS (vetispiradiene synthase) are proposed to play an important role in the cyclization event of F(PP) by deprotonation. In ADS from A. annua, and in a germacrene A synthase (GAS) from Solidago canandensis, the three-amino-acid motif is $\mathrm{Thr}^{401}-\mathrm{Gly}^{402}-\mathrm{Gly}^{403}$. By substituting Gly $^{402}$ in GAS with either Ala or Ser, an accumulation of $\alpha$-humulene (> 40\%) was observed, which in the wild-type GAS only accounts for approximately $2 \%$ of the total product. Substituting the Gly ${ }^{402}$ with Asp or Cys led to an even greater increase in the 1,11-cyclization, whereas more bulky residues started to decrease the 1,11-cyclization activity (Thr and Val) or abolished it altogether (Leu, Ile, and Phe) (Gonzalez et al. 2014). The Thr residue of this motif in ADS plays a crucial role in the deprotonation of the 12/13 proton of amorphadien-11-yl cation (Li et al. 2013; Fang et al. 2017). In all the VvShirazTPS enzymes investigated in our study, Thr and Ser were the corresponding residues of $\mathrm{Thr}^{401}$ and $\mathrm{Thr}^{402}$, respectively, whereas $\mathrm{Thr}^{403}$ is either Ala (VvShirazTPS-Y1 and VvGuaS) or Gly (in all the others). Changing Thr to Ser is likely to have only minor impacts on the specificity in the formation of either 1,10- or 1,11-cyclization products. For instance, changing Thr to Ser in VvGauS did not have large implications on an aberrant product formation (Drew et al. 2016). However, a change like this could have an impact on the catalytic efficiency (Fang et al. 2017). In ZmTPS4 and ZmTPS5 from Z. mays, Ala ${ }^{409}$ and Gly ${ }^{409}$ correspond to $\mathrm{Thr}^{402}$ in TEAS, respectively. $\mathrm{Thr}^{410}$ in ZmTPS4 (Ala ${ }^{410}$ in ZmTPS5) also plays an important role for substrate conversion (Köllner et al. 2004). Pro $^{463}$ in VvShirazTPS-Y2 is in the immediate start of helix G2, a position occupied by Ser in all the other investigated VvShirazTPS sequences. Pro is seldom found in $\alpha$-helices because it distorts their structure (Madison 1977; Kumeta and Ito 2010). However, the Pro in the VvShirazTPS-Y2 enzyme may not have a large impact on the structure since it is in the beginning of the helix. Proline also is present within helix G2 of VvShirazTPS27 and may contribute to a change in the conformation.

Helix H2, aa 440-452

Cys $^{440}$ in TEAS [which is Ser $^{442}$ in GAS, (Gonzalez et al. 2014)] potentially cooperates with $\mathrm{Thr}^{402}$ in the cyclization activity (i.e., 1,10 vs. 1,11). Whether the residue is Ser or Cys could have an impact on the 1,10- and 1,11-cyclization (Yoshikuni et al. 2006; Gonzalez et al. 2014). In VvShirazTPS27 the residue is Ser, like for $\gamma$-humulene synthase from Abiens grandis (Yoshikuni et al. 2006). In VvShirazTPS-Y1, -Y2, and -26 the residue is Cys. Interestingly, the 
equivalent residue in VvShirazTPS07 is Ala, which biosynthesizes germacrene D (and ylangene). This Ala residue is shared among many of the other germacrene and caryophyllene synthases from $V$. vinifera, which indicates that the $\mathrm{Cys}^{440}$ hinders the formation of the $(E, E)$ germacradienyl cation.

$\mathrm{Asn}^{459}$ in helix $\mathrm{H} 2$ is different for the VvShirazTPS-Y2 enzyme compared to the other investigated VvShirazTPS enzymes, in which the corresponding residue is Asp (Asp ${ }^{444}$ in TEAS). Therefore, a polar, uncharged sidechain is present in the VvShirazTPS-Y2 compared to a negative charge, present in the other enzymes. Further, VvShirazTPS-Y2 has a proline residue ( $\mathrm{Thr}^{448}$ in TEAS) where the other terpene synthases have a serine residue. These two residues are part of the DSE/NTE motif (Fig. 2), which is involved in coordinating magnesium ions and thus in the expulsion of the pyrophosphate moiety. Even small changes to this motif can modify the product array (Christianson 2017). The proline residue in VvShirazTPS-Y2 may potentially give more room for the Asn residue (Fig. 3) and water may play an active role instead of serine (Zhou and Peters 2009).

In ZmTPS4 and ZmTPS5 from Z. mays, $\mathrm{Val}^{455}$ and $\mathrm{Glu}^{455}$ (corresponding to $\mathrm{Ala}^{447}$ in TEAS) play an important role in the isomerization to nerolidyl diphosphate relative to the rate of deprotonation of the farnesyl cation (Köllner et al. 2004). In VvShirazTPS07 (Fig. 3), this residue of helix $\mathrm{H} 2$ corresponds to $\mathrm{Lys}^{456}$ (positive charge), whereas in all the other investigated VvShirazTPS enzymes this residue corresponds to Val (smaller, hydrophobic).

Helix $J$ and the J-K loop, aa 512-519 and 520-529

Together with $\mathrm{Thr}^{402}$ in TEAS (in helix G1), $\mathrm{Val}^{516}$ likely plays a crucial role in the product formation. Substituting both residues (TEAS T402S V516I) transmutes TEAS into a 'synthetic' epi-eremophilene synthase (Greenhagen et al. 2006). In the VvShirazTPS enzymes of the present study, the equivalent residue to $\mathrm{Val}^{516}$ is Ile in VvShirazTPS-Y1, -Y2, and -26, Met in -27, and Ala in -07. The corresponding residue is $\mathrm{Val}^{530}$ in VvGuaS. This is interesting, as the site-directed mutation from V530M of VvGauS in Drew et al. (2016) showed a clear change in the product formation shifting from a 5,7-bicyclic carbon skeleton to a 6,6-bicyclic carbon skeleton. This is by Drew et al. (2016) explained by an addition of a lone-pair electrons on Met, which could be involved in the stabilization of the carbonation intermediate. The primary products of the VvShirazTPS27 (that also has Met in that position) are caryophyllenes that structurally are not that similar to selina-4,11-diene, $\alpha$-selinene, epi- $\alpha$-selinene, and intermedeol (that are products of VvGauS). The first cyclization is here a 1,11-cyclization and 
not 1,10 as for $V v G u a S$. Thus, we get the most stable 1,11-carbocations, namely $\alpha$-humulene and $\beta$-caryophyllene, with the $\beta$-caryophyllene being the most stable. Met $^{526}$ in VvShirazTPS27 could still play an active role to stabilize the carbocation but maybe other differences, like Ser ${ }^{450}$ in helix H2, have a key influence on the 1,11 rather than on the 1,10. This residue corresponds to $\mathrm{Cys}^{440}$ in TEAS as described above.

$\mathrm{Asp}^{525}$ in TEAS is well positioned to abstract a proton from C13 (Starks et al. 1997). The equivalent residue is present in all five investigated VvShirazTPS enzymes. $\mathrm{Tyr}^{520}$ in TEAS also constitutes an important first-tier residue of the active site. In TEAS, this tyrosine residue, together with $\mathrm{Asp}^{444}$ and $\mathrm{Asp}^{525}$, in helix G2 and the J-K loop, respectively, could donate a proton to the C6 position of F(PP) (Starks et al. 1997) and perform the protonation of the product. A Y520F mutant of TEAS only generates germacrene A and confirm this position's important role in protonation of the product (Rising et al. 2000). This position was also studied in ZmTPS4 and ZmTPS5 from Z. mays. Here, $\mathrm{Tyr}^{528}$ is the corresponding residue and is identified to have a prominent position in the active site of ZmTPS4 and mutagenesis confirms the importance of this site to lead to a neutral product (Köllner et al. 2006). Asp ${ }^{532}$ was also identified to have an important role in ZmTPS4, and ZmTPS4 appears to require a dyad of $\mathrm{Tyr}^{528}$ and $\mathrm{Asp}^{532}$ to form the (Z,E)-FPP intermediate (Köllner et al. 2006). The influence of this position for protonation was also confirmed in a mutagenic study of the ZmEDS (Liang et al. 2018). As all the VvShirazTPS also have a $\mathrm{Tyr}^{520}$ in this position, it is likely that the functionality is similar to that observed by others. Unlike the investigated VvShirazTPS enzymes, TEAS has an additional amino acid (i.e., Leu ${ }^{524}$ ) just prior to the proton-abstracting $\mathrm{Asp}^{525}$ residue. This may comparatively influence the location of the Asp residue in the $V$. vinifera terpene synthases and how this residue interacts with the farnesyl and/or nerolidyl cations, though this needs to be confirmed by further studies.

As mentioned above, $\operatorname{Tyr}^{527}$ in TEAS together with $\operatorname{Trp}^{273}$ are capable of engaging in cation- $\pi$ interactions. For all the VvShirazTPS enzymes, the corresponding residue is also tyrosine, thus likely playing the same or a very similar role in these enzymes as $\mathrm{Tyr}^{527}$ in TEAS.

\section{Important residues outside the active site}

Only a relatively small number of residues are crucial for the catalytic product formation of TPS enzymes (Fang et al. 2017). In our five characterized $V$. vinifera terpene synthases, activesite residues in helices G1, G2, and H2 seem to constitute much of the difference. However, it is likely that other residues outside the active site contribute to the catalytic product formation 
(Greenhagen et al. 2006; Köllner et al. 2006; González et al. 2016). For instance, the germacrene D synthase VvGwGerD (Martin et al. 2010) and VvShirazTPS07 of the present study are sequentially very similar, differing only in eight locations of which none are in the active site, but with rather different product formations (i.e., 94\% vs. 20\% germacrene D production, respectively). Residues outside the active site are thus likely to play an important role here. For instance, residues in helix 4 of the $\mathrm{N}$-terminal domain appeared important for the functionality of some sesquiterpene synthases from Aquilaria crassna (Kumeta and Ito 2010) and a molecular dynamic study of TEAS points to the N-terminal domain in facilitating the stability of helix $\mathrm{K}$ and the ordering of the J-K loop (Zhang et al. 2016). However, characterization in planta or using microorganisms may also be a contributing factor to this observed difference in germacrene D production (Salvagnin et al. 2016) and the heat in the GC-MS may also have an impact (Andersen et al. 2015).

\section{Combined picture of the five characterized VvShirazTPS enzymes}

VvShirazTPS26 is a multi-product synthase producing among others, $\alpha$-cubebene, $\alpha$-copaene, $(+)$ - $\delta$-cadinene, probably via 1,10-cyclization of the nerolidyl cation (Fig. 1). Nearly half of all characterized terpene synthases are multi-product synthases (Degenhardt et al. 2009). VvShirazTPS27 on the other hand, produces almost entirely $\beta$-caryophyllene (Fig. 1 and Table 1) and the less stable $\alpha$-humulene. The main product of VvShirazTPS-Y2 is also caryophyllenes ( 54\%) probably via 1,11-cyclization of the farnesyl cation, but in addition to this, this synthase also produces $\alpha$-cubebene and $\beta$-cubebene ( 37\%) probably via 1,10cyclization of the nerolidyl cation. However, VvShirazTPS27 has a much higher sequence identity (i.e., 92\%) to VvShirazTPS-Y1, which produces a wide range of compounds with a 6,6-bicyclic carbon skeleton, mostly ( + )- $\delta$-cadinene (Fig. 1 and Table 1$)$. The main differences in residue compositions of the active sites between these two enzymes come down to Pro for VvShirazTPS27 vs. Ser for VvShirazTPS-Y1 in helix G2, Ser vs. Cys in helix H2, and Met vs. Ile in helix J (Fig. 2 and Fig. 3). Further investigations, for example using site-directed mutagenesis, are needed to determine the role of these residue differences on product profiles. However, general features in the differentiation between multi-product TPSs and those that biosynthesize almost exclusively one product are hard to find (Degenhardt et al. 2009).

It is of great interest to identify key residues in the first cyclization event (e.g., 1,10- vs. 1,11cyclization), since this knowledge provides insights into evolution, guiding optimization of terpene synthases and/or creating terpene synthases with novel properties (McAndrew et al. 
2011; Gonzalez et al. 2014; Fang et al. 2017). In ADS of A. annua, $\operatorname{Thr}^{296}$ ( $\operatorname{Ser}^{298}$ in TEAS) in helix D has been identified to play a key role in the first cyclization (1,6-cyclization of the cisnerolidyl cation) and the residues Leu ${ }^{374}$ (helix F), Leu ${ }^{404}$ (helix G2), Leu ${ }^{405}$ (helix G2), and Gly $^{439}$ (helix H2) collectively guide the second ring closure (Fang et al. 2017). This is quite different from TEAS, where $\mathrm{Thr}^{401}-\mathrm{Thr}^{402}-\mathrm{Thr}^{403}$ in helix G1 are likely key residues in the first cyclization event. Here, the neutral germacrene D intermediate is produced (Starks et al. 1997). The corresponding amino-acid triad (Thr-Gly-Gly) in GAS, especially the second residue, as mentioned above, appear important to whether a 1,10- or a 1,11-ring closure of the farnesyl cation occurs (Gonzalez et al. 2014). In all our investigated terpene synthases, the second residue in this triad is serine, and the product profiles resemble those of TEAS and GAS. The interaction of this serine residue (in the middle of helix G1) with Cys/Ala/Ser (in the start of helix H2) and Val/Met/Ala/Ile (a third into helix J) seems to correspond with findings from TEAS and GAS and can be part of the first cyclization event. These three neighboring residues on three different helices are likely positioned near the third double bond of the FPP (Online Resource 3).

Not only are the chemical properties of the active-site residues important to consider, but also their size. Relative to the other VvShirazTPS enzymes, the active-site volume of VvShirazTPS26 seems smaller (Fig. 3). However, the active-site volumes of the investigated TPSs are not that different and configuration of the J-K loop in the homology models may also contribute to the volume differences and our models are not conclusive here.

\section{Conclusions}

Here we have characterized five terpene synthases from Vitis vinifera cv. Shiraz, two of which were previously uncharacterized. VvShirazTPS-Y1 is a cadinene synthase, and VvShirazTPSY2 is caryophyllene/cubebene synthase. The interactions between the amino acids in helices $\mathrm{G} 1, \mathrm{H} 2$, and $\mathrm{J}$ were identified as the main differences that influence the first cyclization event for the five TPSs, where the second amino acid in the triad was a serine (helix G1) that interacts with Cys/Ala/Ser (helix H2) and Val/Met/Ala/Ile (helix J). These interactions correspond with the findings from TEAS and GAS.

We still do not know much about the relative influence of each amino acid to the product formations of single- and multi-product synthases. As for many other investigated TPS enzymes, only a few residues can account for radical changes in product formation, and further 
crystallography studies based on proteins isolated from plants is needed to further explore this field.

\section{Author contribution}

HTS and DPD conceived and designed research. DPD and CS conducted the in planta and GCMS experiments and BD performed the in silico analysis. BD, DPD, CMF and HTS analyzed data. BD, DPD and HTS wrote the manuscript. All authors read and approved the manuscript. Conflict of Interest: The authors declare that they have no conflict of interest.

\section{Acknowledgements}

This research was supported by the European Union Seventh Framework Programme (FP7/2007-2013) under grant agreement 275422, which supported a Marie Curie International Outgoing Fellowship (DPD). The Danish Strategic Research Council Grant "SPOTLight” supported the work of HTS. 


\section{References}

Andersen T, Cozzi F, Simonsen HT (2015) Optimization of biochemical screening methods for volatile and unstable sesquiterpenoids using HS-SPME-GC-MS. Chromatography 2:277292. doi: 10.3390/chromatography2020277

Bach SS, Bassard J-É, Andersen-Ranberg J, et al (2014) High throughput testing of terpenoid biosynthesis candidate genes using transient expression in Nicotiana benthamiana. In: Rodríguez-Concepción M (ed) Plant Isoprenoids, 1153rd edn. Springer, New York

Battilana J, Emanuelli F, Gambino G, et al (2011) Functional effect of grapevine 1-deoxy-Dxylulose 5-phosphate synthase substitution K284N on Muscat flavour formation. J Exp Bot err231. doi: https://doi.org/10.1093/jxb/err231

Chen F, Tholl D, Bohlmann J, Pichersky E (2011) The family of terpene synthases in plants: a mid-size family of genes for specialized metabolism that is highly diversified throughout the kingdom. Plant J 66:212-229. doi: 10.1111/j.1365-313X.2011.04520.x

Chen VB, Arendall WB, Headd JJ, et al (2010) MolProbity: all-atom structure validation for macromolecular crystallography. Acta Crystallogr Sect D Biol Crystallogr 66:12-21. doi: 10.1107/S0907444909042073

Christianson DW (2017) Structural and chemical biology of terpenoid cyclases. Chem Rev 117:11570-11648. doi: 10.1021/acs.chemrev.7b00287

Coelho E, Rocha SM, Delgadillo I, Coimbra MA (2006) Headspace-SPME applied to varietal volatile components evolution during Vitis vinifera L. cv. "Baga” ripening. Anal Chim Acta 563:204-214. doi: 10.1016/j.aca.2005.11.018

Davis EM, Croteau R (2000) Cyclization Enzymes in the Biosynthesis of Monoterpenes, Sesquiterpenes, and Diterpenes. In: Leeper FJ, Vedera JC (eds) Biosynthesis. pp 53-95

Degenhardt J, Köllner TG, Gershenzon J (2009) Monoterpene and sesquiterpene synthases and the origin of terpene skeletal diversity in plants. Phytochemistry 70:1621-1637. doi: 10.1016/j.phytochem.2009.07.030

Dougherty DA (1996) Cation- $\pi$ interactions in chemistry and biology: a new view of benzene, Phe, Tyr, and Trp. Science 271:163-168. doi: 10.1126/science.271.5246.163

Drew DP, Andersen TB, Sweetman C, et al (2016) Two key polymorphisms in a newly discovered allele of the Vitis vinifera TPS24 gene are responsible for the production of the rotundone precursor $\alpha$-guaiene. J Exp Bot 67:799-808. doi: 10.1093/jxb/erv491 
Drew DP, Dueholm B, Weitzel C, et al (2013) Transcriptome analysis of Thapsia laciniata Rouy provides insights into terpenoid biosynthesis and diversity in Apiaceae. Int J Mol Sci 14:9080-9098. doi: 10.3390/ijms14059080

Drew DP, Rasmussen SK, Avato P, Simonsen HT (2012) A comparison of headspace solidphase microextraction and classic hydrodistillation for the identification of volatile constituents from Thapsia spp. provides insights into guaianolide biosynthesis in Apiaceae. Phytochem Anal 23:44-51. doi: 10.1002/pca.1323

Dunlevy JD, Kalua CM, Keyzers RA, Boss PK (2009) The Production of Flavour \& Aroma Compounds in Grape Berries. In: Grapevine Molecular Physiology \& Biotechnology. Springer, pp 293-340

Durrant JD, De Oliveira CAF, McCammon JA (2011) POVME: An algorithm for measuring binding-pocket volumes. J Mol Graph Model 29:773-776. doi: 10.1016/j.jmgm.2010.10.007

Durrant JD, Votapka L, Sørensen J, Amaro RE (2014) POVME 2.0: An enhanced tool for determining pocket shape and volume characteristics. J Chem Theory Comput 10:5047-5056. doi: 10.1021/ct500381c

Dziadas M, Jeleń HH (2010) Analysis of terpenes in white wines using SPE-SPME-GC/MS approach. Anal Chim Acta 677:43-49. doi: 10.1016/j.aca.2010.06.035

Fang X, Li J-X, Huang J-Q, et al (2017) Systematic identification of functional residues of Artemisia annua amorpha-4,11-diene synthase. Biochem J 474:2191-2202. doi: 10.1042/BCJ20170060

Gao Y, Honzatko RB, Peters RJ (2012) Terpenoid synthase structures: a so far incomplete view of complex catalysis. Nat Prod Rep 29:1153. doi: 10.1039/c2np20059g

González V, Grundy DJ, Faraldos JA, Allemann RK (2016) The amino-terminal segment in the $\beta$-domain of $\delta$-cadinene synthase is essential for catalysis. Org Biomol Chem 14:74517454. doi: 10.1039/C6OB01398H

Gonzalez V, Touchet S, Grundy DJ, et al (2014) Evolutionary and mechanistic insights from the reconstruction of $\alpha$-humulene synthases from a modern (+)-germacrene A synthase. $\mathrm{J}$ Am Chem Soc 136:14505-14512. doi: 10.1021/ja5066366

Greenhagen BT, O’Maille PE, Noel JP, Chappell J (2006) Identifying and manipulating structural determinates linking catalytic specificities in terpene synthases. Proc Natl Acad Sci 103:9826-9831. doi: 10.1073/pnas.0601605103 
Ikram NKBK, Zhan X, Pan X-W, et al (2015) Stable heterologous expression of biologically active terpenoids in green plant cells. Front Plant Sci 6:129. doi: 10.3389/fpls.2015.00129

Ilc T, Halter D, Miesch L, et al (2017) A grapevine cytochrome P450 generates the precursor of wine lactone, a key odorant in wine. New Phytol 213:264-274. doi: 10.1111/nph.14139

Kalua CM, Boss PK (2010) Comparison of major volatile compounds from Riesling and Cabernet Sauvignon grapes (Vitis vinifera L.) from fruitset to harvest. Aust J Grape Wine Res 16:337-348. doi: 10.1111/j.1755-0238.2010.00096.x

Köllner TG, Maille PEO, Gatto N, et al (2006) Two pockets in the active site of maize sesquiterpene synthase TPS4 carry out sequential parts of the reaction scheme resulting in multiple products. Arch Biochem Biophys 448:83-92. doi: 10.1016/j.abb.2005.10.011

Köllner TG, Schnee C, Gershenzon J, Degenhardt J (2004) The variability of sesquiterpenes emitted from two Zea mays cultivars is controlled by allelic variation of two terpene synthase genes encoding stereoselective multiple product enzymes. Plant Cell 16:1115-1131. doi: 10.1105/tpc.019877.tive

Kumeta Y, Ito M (2010) Characterization of $\delta$-guaiene synthases from cultured cells of Aquilaria, responsible for the formation of the sesquiterpenes in agarwood. Plant Physiol 154:1998-2007. doi: 10.1104/pp.110.161828

Li J-X, Fang X, Zhao Q, et al (2013) Rational engineering of plasticity residues of sesquiterpene synthases from Artemisia annua: product specificity and catalytic efficiency. Biochem J 451:417-426. doi: 10.1042/BJ20130041

Liang J, Liu J, Brown R, et al (2018) Direct production of di-hydroxylated sesquiterpenoids by a maize terpene synthase. Plant J. doi: 10.1111/tpj.13901

Lund ST, Bohlmann J (2006) The molecular basis for wine grape quality - a volatile subject. Science 311:804-805. doi: 10.1126/science.1118962

Lüthy R, Bowie JU, Eisenberg D (1992) Assessment of protein models with three-dimensional profiles. Nature 356:83-85. doi: 10.1038/356083a0

Madison V (1977) Flexibility of the pyrrolidine ring in proline peptides. Biopolymers 16:26712692. doi: 10.1002/bip.1977.360161208

Martin DM, Aubourg S, Schouwey MB, et al (2010) Functional annotation, genome organization and phylogeny of the grapevine (Vitis vinifera) terpene synthase gene family 
based on genome assembly, FLcDNA cloning, and enzyme assays. BMC Plant Biol 10:226. doi: 10.1186/1471-2229-10-226

Martin DM, Bohlmann J (2004) Identification of Vitis vinifera (-)- $\alpha$-terpineol synthase by in silico screening of full-length cDNA ESTs and functional characterization of recombinant terpene synthase. Phytochemistry 65:1223-1229. doi: 10.1016/j.phytochem.2004.03.018

Martin DM, Chiang A, Jo STL, et al (2012) Biosynthesis of wine aroma: transcript profiles of hydroxymethylbutenyl diphosphate reductase, geranyl diphosphate synthase, and linalool/nerolidol synthase parallel monoterpenol glycoside accumulation in Gewürztraminer grapes. Planta 236:919-929. doi: 10.1007/s00425-012-1704-0

Martin DM, Toub O, Chiang A, et al (2009) The bouquet of grapevine (Vitis vinifera L. cV. Cabernet Sauvignon) flowers arises from the biosynthesis of sesquiterpene volatiles in pollen grains. Proc Natl Acad Sci 106:7245-7250. doi: 10.1073/pnas.0901387106

Matarese F, Cuzzola A, Scalabrelli G, D’Onofrio C (2014) Expression of terpene synthase genes associated with the formation of volatiles in different organs of Vitis vinifera. Phytochemistry 105:12-24. doi: 10.1016/j.phytochem.2014.06.007

Matarese F, Scalabrelli G, D’Onofrio C (2013) Analysis of the expression of terpene synthase genes in relation to aroma content in two aromatic Vitis vinifera varieties. Funct Plant Biol 40:552-565. doi: 10.1071/FP12326

May B, Wüst M (2012) Temporal development of sesquiterpene hydrocarbon profiles of different grape varieties during ripening. Flavour Fragr J 27:280-285. doi: 10.1002/ffj.3096

McAndrew RP, Peralta-Yahya PP, DeGiovanni A, et al (2011) Structure of a three-domain sesquiterpene synthase: a prospective target for advanced biofuels production. Structure 19:1876-1884

Meng EC, Pettersen EF, Couch GS, et al (2006) Tools for integrated sequence-structure analysis with UCSF Chimera. BMC Bioinformatics 7:1-10. doi: 10.1186/1471-2105-7-339

Nour-Eldin HH, Hansen BG, Nørholm MHH, et al (2006) Advancing uracil-excision based cloning towards an ideal technique for cloning PCR fragments. Nucleic Acids Res 34:. doi: 10.1093/nar/gkl635

Paré PW, Tumlinson JH (1999) Plant volatiles as a defense against insect herbivores. Plant Physiol 121:325-332. doi: 10.1104/pp.121.2.325 
Pettersen EF, Goddard TD, Huang CC, et al (2004) UCSF Chimera - A visualization system for exploratory research and analysis. J Comput Chem 25:1605-1612. doi: 10.1002/jcc.20084

Rising KA, Starks CM, Noel JP, Chappell J (2000) Demonstration of germacrene A as an intermediate in 5-Epi-aristolochene synthase catalysis. J Am Chem Soc 122:1861-1866. doi: 10.1021/ja993584h

Robinson AL, Boss PK, Solomon PS, et al (2014) Origins of grape and wine aroma. Part 1. Chemical components and viticultural impacts. Am J Enol Vitic 65:1-24. doi: 10.5344/ajev.2013.12070

Rupasinghe S, Schuler MA (2006) Homology modeling of plant cytochrome P450s. Phytochem Rev 5:473-505. doi: 10.1007/s11101-006-9028-y

Sali A, Blundell TL (1993) Comparative protein modeling by satisfaction of spatial restraint. J Mol Biol 234:779-815. doi: 10.1006/jmbi.1993.1626

Salvagnin U, Carlin S, Angeli S, et al (2016) Homologous and heterologous expression of grapevine E-( $\beta$ )-caryophyllene synthase (VvGwECar2). Phytochemistry 131:76-83. doi: 10.1016/j.phytochem.2016.08.002

Sánchez-Palomo E, Diaz-Maroto MC, Perez-Coello MS (2005) Rapid determination of volatile compounds in grapes by HS-SPME coupled with GC-MS. Talanta 66:1152-1157. doi: 10.1016/j.talanta.2005.01.015

Starks CM, Back K, Chappell J, Noel JP (1997) Structural basis for cyclic terpene biosynthesis by tobacco 5-epi-aristolochene synthase. Science 277:1815-1820. doi: 10.1126/science.277.5333.1815

Sweetman C, Wong DCJ, Ford CM, Drew DP (2012) Transcriptome analysis at four developmental stages of grape berry (Vitis vinifera cv. Shiraz) provides insights into regulated and coordinated gene expression. BMC Genomics 13:1. doi: 10.1186/1471-2164-13-691

Trott O, Olson AJ (2010) AutoDock Vina: improving the speed and accuracy of docking with a new scoring function, efficient optimization, and multithreading. J Comput Chem 31:455461. doi: $10.1002 / j c c$

Vilanova M, Sieiro C (2006) Determination of free and bound terpene compounds in Albarino wine. J Food Compos Anal 19:694-697. doi: 10.1016/j.jfca.2005.10.002

Webb B, Sali A (2016) Comparative protein structure modeling using MODELLER. Curr 
Protoc Protein Sci 86:2.9.1-2.9.37. doi: doi.org/10.1002/cpps.20

Weitzel C, Simonsen HT (2015) Cytochrome P450-enzymes involved in the biosynthesis of mono- and sesquiterpenes. Phytochem Rev 14:7-24. doi: 10.1007/s11101-013-9280-x

Wood C, Siebert TE, Parker M, et al (2008) From wine to pepper: rotundone, an obscure sesquiterpene, is a potent spicy aroma compound. J Agric Food Chem 56:3738-3744. doi: $10.1021 / \mathrm{jf} 800183 \mathrm{k}$

Yang K, Monafared RS, Wang H, et al (2015) The activity of the artemisinic aldehyde $\Delta 11(13)$ reductase promoter is important for artemisinin yield in different chemotypes of Artemisia annua L. Plant Mol Biol 88:325-340. doi: 10.1007/s11103-015-0284-3

Yoshikuni Y, Ferrin TE, Keasling JD (2006) Designed divergent evolution of enzyme function. Nature 440:1078-1082. doi: 10.1038/nature04607

Zhan X, Zhang Y-H, Chen D-F, Simonsen HT (2014) Metabolic engineering of the moss Physcomitrella patens to produce the sesquiterpenoids patchoulol and $\alpha / \beta$-santalene. Front Plant Sci 5:636. doi: 10.3389/fpls.2014.00636

Zhang F, Chen N, Wu R (2016) Molecular dynamics simulations elucidate conformational dynamics responsible for the cyclization reaction in TEAS. J Chem Inf Model 56:877-885. doi: 10.1021/acs.jcim.6b00091

Zhou K, Peters RJ (2009) Investigating the conservation pattern of a putative second terpene synthase divalent metal binding motif in plants. Phytochemistry 70:366-369. doi: 10.1016/j.phytochem.2008.12.022 


\section{Tables}

Table 1. Identified sesquiterpenoids produced by the five $V$. vinefera terpene synthases. The area percentage is calculated based on the total integrated area of the run.

\begin{tabular}{|c|c|c|c|c|c|c|c|c|c|c|c|c|c|c|c|c|c|}
\hline \multirow[b]{2}{*}{ Rt Compound } & \multirow{2}{*}{\multicolumn{2}{|c|}{ Litt. RI RI }} & \multirow{2}{*}{$\begin{array}{l}\text { TPS07, } \\
\text { synthase } \\
\text { Library } \\
\text { hit \% }\end{array}$} & \multicolumn{3}{|c|}{ Ylangene/germacrene TPS26, } & \multicolumn{2}{|c|}{ Multiple-product synthas } & \multicolumn{3}{|c|}{ se TPS27, Isocaryophyllene syntha } & \multicolumn{3}{|c|}{ se TPS-Y1, Cadinene synthase } & \multicolumn{3}{|c|}{ TPS-Y2, Caryophyllene synthase } \\
\hline & & & & TIC Area & $\begin{array}{l}\% \\
\text { Area }\end{array}$ & $\begin{array}{l}\text { of Library } \\
\text { hit \% }\end{array}$ & TIC Area & $\begin{array}{l}\% \\
\text { Area }\end{array}$ & $\begin{array}{c}\text { of Library } \\
\text { hit \% }\end{array}$ & TIC Area & $\begin{array}{l}\% \\
\text { Area }\end{array}$ & $\begin{array}{l}\text { of Library } \\
\text { hit \% }\end{array}$ & TIC Area & $\begin{array}{l}\% \\
\text { Area }\end{array}$ & $\begin{array}{l}\text { of Library } \\
\text { hit \% }\end{array}$ & TIC Area & $\begin{array}{l}\% \\
\text { Area }\end{array}$ \\
\hline $12.38 \alpha$-cubebene & $\begin{array}{l}1347- \\
1353\end{array}$ & 1348 & & & & $97 \%$ & 39469000 & $27 \%$ & & & & $95 \%$ & 2164000 & $5 \%$ & $97 \%$ & 37058000 & $14 \%$ \\
\hline 12.78 ylangene & $\begin{array}{l}1359- \\
1372\end{array}$ & 1369 & $95 \%$ & 16430000 & $35 \%$ & & & & & & & $93 \%$ & 1771000 & $4 \%$ & & & \\
\hline $12.88 \alpha$-copaene & $\begin{array}{l}1372- \\
1376\end{array}$ & 1374 & & & & $96 \%$ & 30890000 & $21 \%$ & & & & $95 \%$ & 1880000 & $4 \%$ & $95 \%$ & 11487000 & $4 \%$ \\
\hline $13.14 \beta$-cubebene & $\begin{array}{l}1381- \\
1390\end{array}$ & 1388 & & & & $91 \%$ & 3988000 & $3 \%$ & & & & & & & $92 \%$ & 61790000 & $23 \%$ \\
\hline $13.50 \alpha$-gurjunene & $\begin{array}{l}1402- \\
1411\end{array}$ & 1407 & & & & $97 \%$ & 9830000 & $7 \%$ & & & & & & & & & \\
\hline 13.66 aromadendrene & $\begin{array}{l}1407- \\
1440\end{array}$ & 1416 & $93 \%$ & 3213000 & $7 \%$ & & & & & & & $92 \%$ & 2735000 & $6 \%$ & & & \\
\hline 13.67 isocaryophyllene & $\begin{array}{l}1404- \\
1428\end{array}$ & $\begin{array}{l}1417- \\
1419\end{array}$ & & & & $96 \%$ & 5375000 & $4 \%$ & $96 \%$ & 278868000 & $82 \%$ & & & & $96 \%$ & 85511000 & $31 \%$ \\
\hline $14.06 \alpha$-guaiene & $\begin{array}{l}1428- \\
1457\end{array}$ & 1437 & & & & & & & & & & & & & & & \\
\hline 14.11 aristolene & $\begin{array}{l}1423- \\
1486\end{array}$ & 1440 & $90 \%$ & 2347000 & $5 \%$ & & & & & & & & & & & & \\
\hline 14.21 alloaromadendrene & $\begin{array}{l}1442- \\
1467\end{array}$ & 1446 & $91 \%$ & 2591000 & $5 \%$ & & & & & & & & & & & & \\
\hline $14.31 \alpha$-caryophyllene & $\begin{array}{l}1438- \\
1463\end{array}$ & 1451 & & & & & & & $97 \%$ & 56296000 & $17 \%$ & & & & $97 \%$ & 56536000 & $21 \%$ \\
\hline $14.72 \gamma$-muurolene & $\begin{array}{l}1462- \\
1485\end{array}$ & 1474 & $90 \%$ & 3547000 & $7 \%$ & & & & & & & $92 \%$ & 3547000 & $7 \%$ & & & \\
\hline
\end{tabular}




\begin{tabular}{|c|c|c|c|c|c|c|c|c|c|c|c|c|c|c|c|c|c|}
\hline $14.78 \gamma$-gurjunene & 1479 & 1478 & & & & & & & & & & & & & & & \\
\hline 14.80 (-)-germacrene D & $\begin{array}{l}1477- \\
1483\end{array}$ & 1479 & $97 \%$ & 9694000 & $20 \%$ & & & & & & & $96 \%$ & 3469000 & $7 \%$ & & & \\
\hline 14.95 epiglobulol & $\begin{array}{l}1463- \\
1497\end{array}$ & 1487 & & & & & & & & & & & & & & & \\
\hline 15.04 valencene & $\begin{array}{l}1463- \\
1494\end{array}$ & 1492 & & & & & & & $92 \%$ & 1062000 & $0 \%$ & & 2197000 & $5 \%$ & & & \\
\hline $15.13 \alpha$-muurolene & $\begin{array}{l}1493- \\
1505\end{array}$ & 1498 & & & & $92 \%$ & 1895000 & $1 \%$ & & & & $91 \%$ & 2773000 & $6 \%$ & & & \\
\hline $15.26 \delta$-guaiene & $\begin{array}{l}1500- \\
1515\end{array}$ & 1505 & & & & & & & & & & & & & & & \\
\hline $15.38 \gamma$-cadinene & $\begin{array}{l}1511- \\
1515\end{array}$ & 1512 & $93 \%$ & 2717000 & $6 \%$ & $90 \%$ & 1218000 & $1 \%$ & & & & $94 \%$ & 4577000 & $10 \%$ & & & \\
\hline 15.44 (-)- $\alpha$-panasinsen & 1518 & 1515 & & & & & & & & & & & & & & & \\
\hline $15.53(+)$ - $\delta$-cadinene & $\begin{array}{l}1519- \\
1530\end{array}$ & 1521 & $96 \%$ & 5848000 & $12 \%$ & $96 \%$ & 30159000 & $21 \%$ & $95 \%$ & 2089000 & $1 \%$ & $95 \%$ & 13088000 & $27 \%$ & $95 \%$ & 11577000 & $4 \%$ \\
\hline 15.69 cadina-1,4-diene & $\begin{array}{l}1524- \\
1539\end{array}$ & 1530 & & & & $94 \%$ & 7744000 & $5 \%$ & & & & $94 \%$ & 1255000 & $3 \%$ & $94 \%$ & 4384000 & $2 \%$ \\
\hline 16.55 caryophyllene oxide & $\begin{array}{l}1573- \\
1585\end{array}$ & 1581 & & & & & & & & & & & & & $92 \%$ & 4963000 & $2 \%$ \\
\hline $17.29 \delta$-cadinol & $\begin{array}{l}1618- \\
1646\end{array}$ & 1626 & & & & $82 \%$ & 959000 & $1 \%$ & & & & & & & & & \\
\hline 17.72 pogostol & $\begin{array}{l}1637- \\
1656\end{array}$ & 1653 & & & & & & & & & & & & & & & \\
\hline $17.72 \alpha$-cadinol & $\begin{array}{l}1643- \\
1656\end{array}$ & 1653 & & & & & & & & & & $91 \%$ & 1468000 & $3 \%$ & & & \\
\hline
\end{tabular}




\section{Figures}

Figure 1: Overview of the biochemical steps catalyzed by the characterized enzymes, VvShirazTPS07, VvShirazTPS26, VvShirazTPS27, VvShirazTPS-Y1, and VvShirazTPS-Y2 from Vitis vinefera.

Figure 2: Sequence alignments and identification of important residues in or close to the active sites of the VvShirazTPS homology models based on a TEAS crystal structure (following TEAS residue numbering). Dots denote amino acids that are identical to the template. The DDxxD motif and the DSE/NTE motif in helix D and helix H2, respectively, are framed in blue. Residues framed in red are likely to contribute to the differences in product profiles between the VvShirazTPS enzymes. Residues framed in black are validated as important for substrate conversion in TPSs but are considered less likely to have a large impact on differences in product profiles of the investigated VvShirazTPS enzymes. The two residues of the VvGauS sequence that are framed with dashed lines are those investigated by site-directed mutagenesis in Drew et al. (2016).

Figure 3: Docking of intermediates and volumes of the TPS actives sites. Intermediates in hot

pink are $(Z, E)$-germacradienyl cations, that come from a 1,10-ring closure of the nerolidyl cation. Intermediates in green are cations that come from either a 1,10-ring closure (TPS07 and TPS-Y2) or a 1,11-ring closure (TPS27) of the farnesyl cation. Percentages indicate the products generated from the respective intermediates. Surfaces of the active-site interiors are also presented. Residues of the DDxxD motif and the DSE/NTE motif in helix D and helix H2, respectively, are colored blue. Major differences in active-site residues are colored in red.

\section{Supplementary figures}

Online Resource 1: Product profiles of the five characterized VvShirazTPS enzymes.

Online Resource 2: Full-length sequence alignment of the VvShirazTPS enzymes.

Online Resource 3: Positions of residues Ser, Ser, and Met in VvShirazTPS27 corresponding to residue-locations 402, 440, and 516 in TEAS. Distances from the residues to C10 of FPP are shown. 
A - First closure

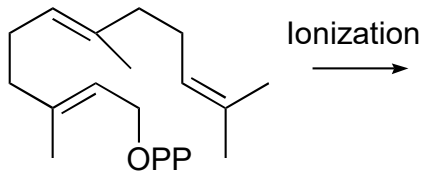

E,E-Farnesyl diphosphate<smiles>CCC[CH-]C=C(C)CCC=C(C)CCC=C(C)C</smiles><smiles>CC1=COCC2CC(C)=CCC2=CCC1</smiles>

Farnesyl cation

Nerolidyl cation

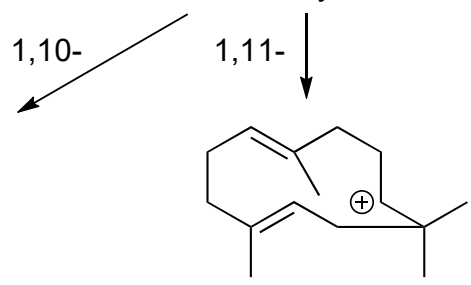<smiles>CCC=C(C)CCC(CC=C(C)C(C)C)C(C)C</smiles>

$(E, E)$-germacradienyl cation

(E,E)-humulyl cation

$(Z, E)$-germacradienyl
D $\mid$ TPS07
C| $\downarrow \begin{aligned} & \text { TPS-Y2 } \\ & \text { TPS27 }\end{aligned}$

B $\mid \begin{aligned} & \text { TPS26, TPS07 } \\ & \text { TPS-Y1, TPS-Y2 }\end{aligned}$<smiles>CC1=C[C@H]2C(C)=CCC(C(C)C)[C@H]2CC1</smiles>

$\alpha$-muurolene<smiles>C=C1CCC(C(C)C)[C@]2(C)C=C(C)CC[C@@H]12</smiles>

$d_{e p r o} \gamma$-muurolene

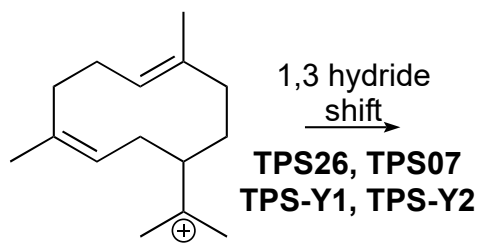

$(Z, E)$-germacradienyl cation<smiles>CC1=C[C-]C(C(C)C)CCC(C)=CCC1</smiles>

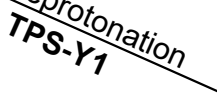

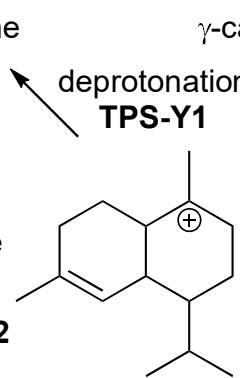

$\gamma$-cadinene<smiles>C=C1CCC(C(C)C)[C@]2(C)C=C(C)CC[C@H]12</smiles>
$\delta$-cadinene

S26, TPS-Y1, TPS-Y2
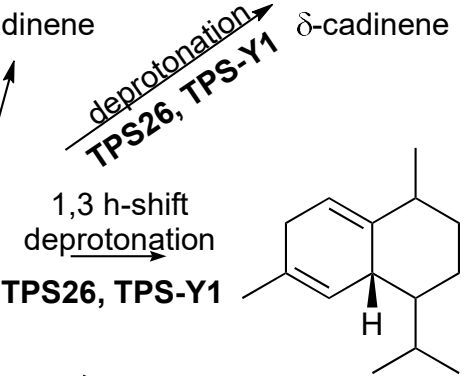<smiles>C=C1CCC23CC1C2C(C(C)C)CCC3C</smiles>

$\beta$-cubebene
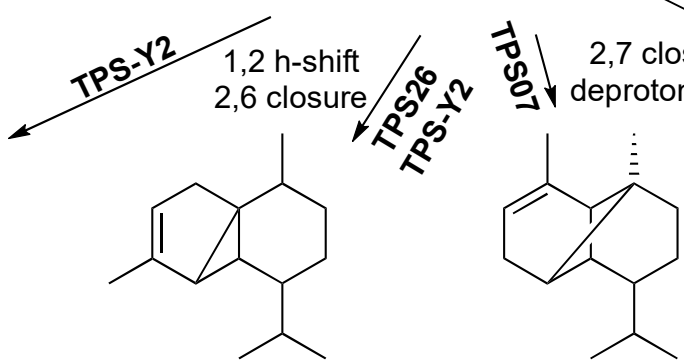

$\mathrm{TP}_{\mathrm{S}_{6}}$

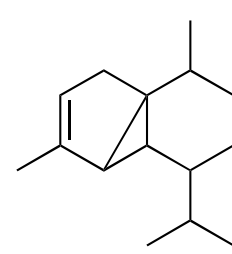

cadina-1,4-diene

$\alpha$-cubebene

$\alpha$-ylangene

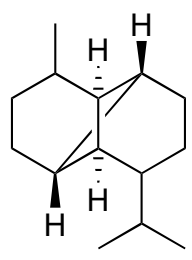

$\alpha$-copaene

\section{C - $(E, E)$-humulyl closure}

\section{and deprotonation}

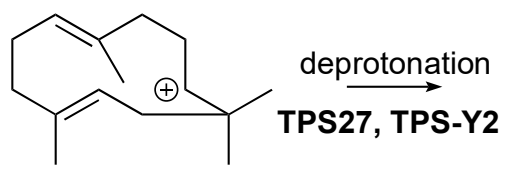

(E,E)-humulyl cation

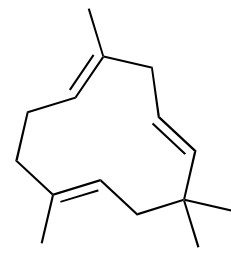

$\alpha$-humulene

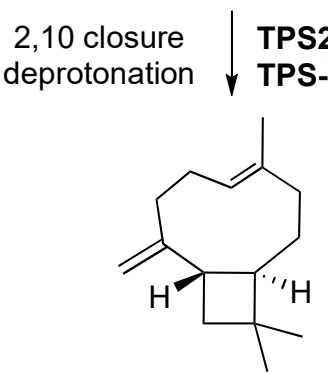

$\beta$-caryophyllene

\section{D - (E,E)-germacradienyl closure and deprotonation}<smiles>CC1=CCCC(C(C)C)CCC1</smiles>

$(E, E)$-germacradienyl cation

\section{1,3 h-shift deprotonation \\ TPS07}<smiles>C=C1/C=C2/C/C(=C\CC1)CCC(C(C)C)/C=C/C2=C</smiles>

germacrene $D$ 


\section{A-C loop and helix $C$}

Helix D

Helix F

Helices $\mathrm{G} 1$ and $\mathrm{G} 2$

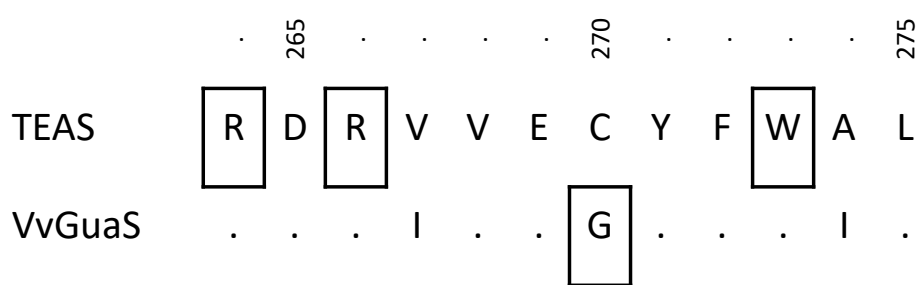

07

26

27

Y1

Y2

\section{Helix H2}

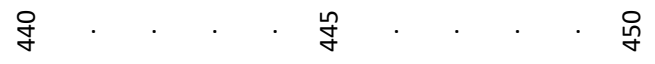

TEAS

\begin{tabular}{|l|l|ll|llll|l|lllll}
\hline$C$ & $R$ & $V$ & I & D & D & $T$ & A & T & Y & E & $V$ & E \\
\hline
\end{tabular}

VvGuaS

07

A . L M $. \quad . \quad I \quad K \quad S \quad H \quad K \quad F$.

26

$L M$. I V S H.F

27

Y1

$S \cdot L M \cdot M V S H K F$

$L M$. . M V S H K F.

$L \quad M \quad N$. M V $P$ H

\section{Helix J and the J-K loop}

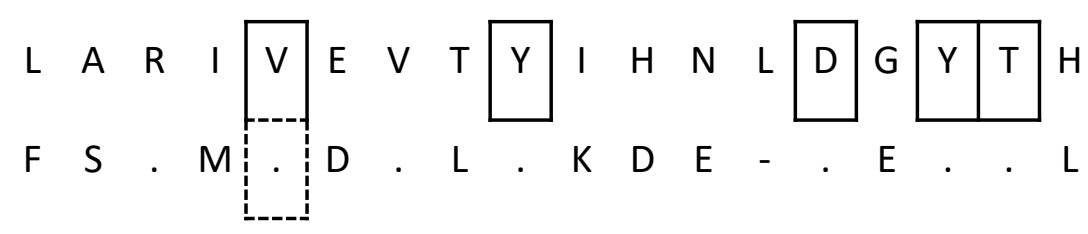

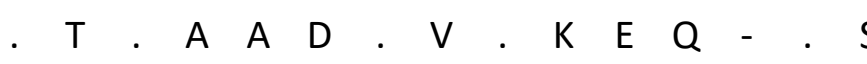

F S . V I D I L .

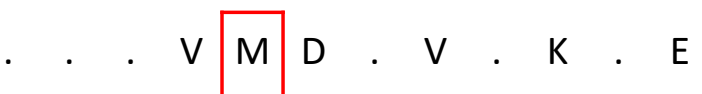

I D

F I D . V . K D E 

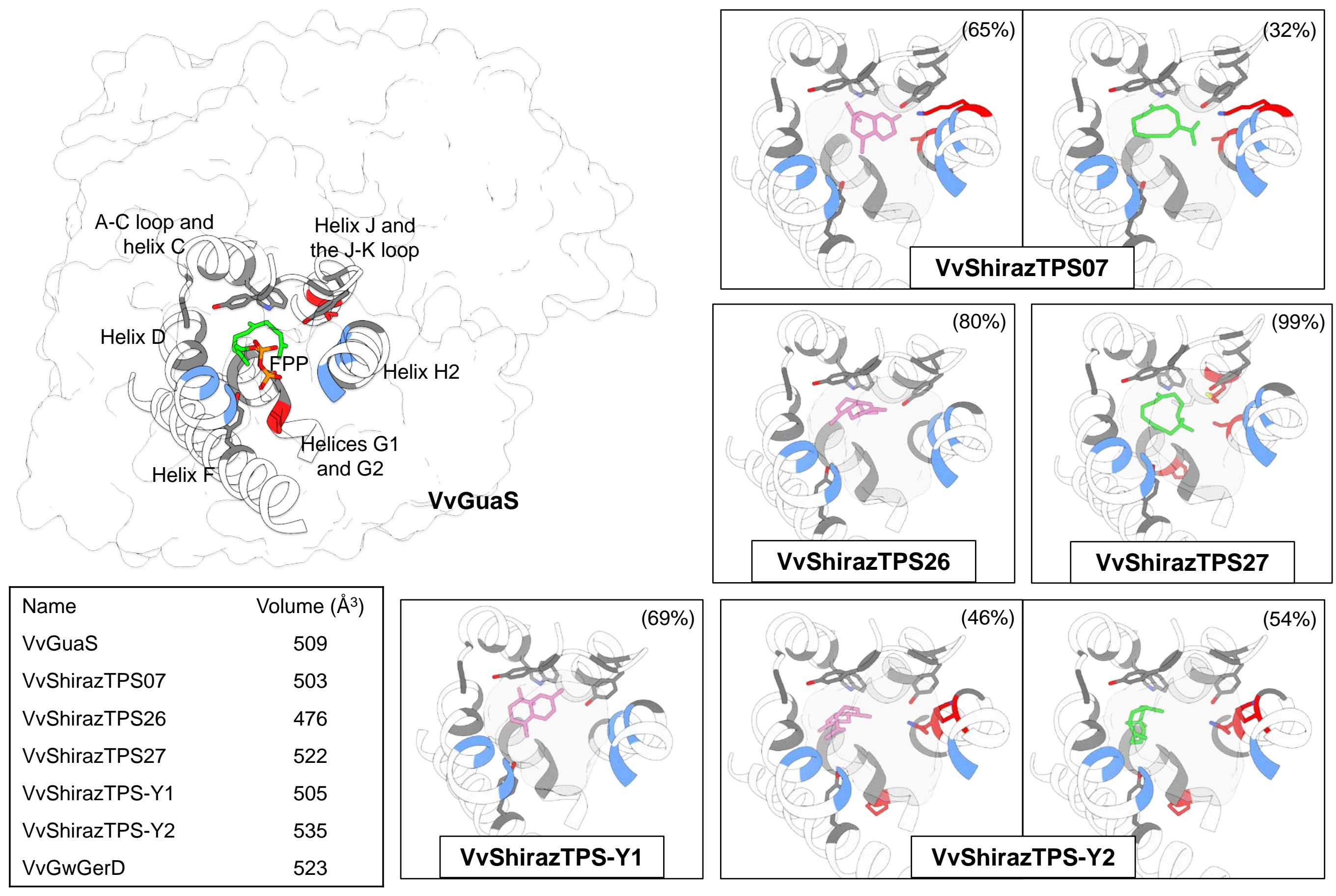

VvGuas

VvShirazTPS26

VvShirazTPS27
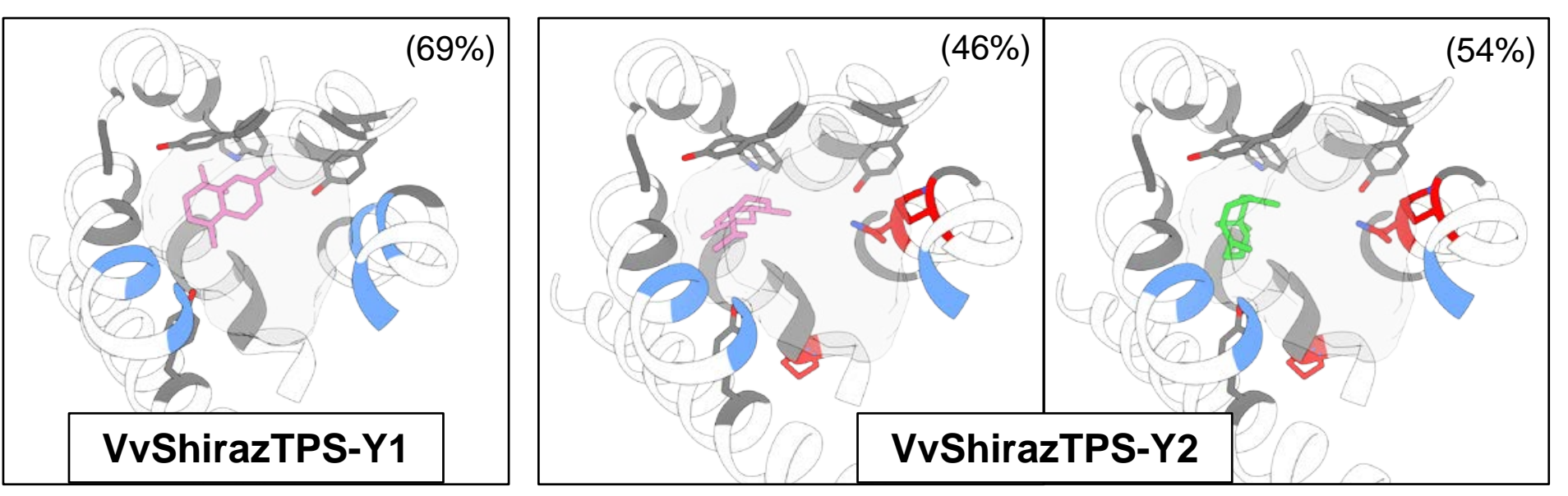